\title{
Virtuelles Seminar zur Fachdidaktik des Rechnungswesenunterrichts: Implementation und erste Erfahrungen
}

Karsten D. Wolf, Jürgen Seifried und Helge Städtler

\section{Ausgangssituation}

Um Schülerinnen und Schülern bei der Bewältigung der zunehmenden Komplexität und Unbestimmtheit im beruflichen Kontext behilflich zu sein, zielen Bildungs- und Qualifizierungsprozesse verstärkt auf "Selbstorganisation" und "Selbstverantwortung". In diesem Zusammenhang stellt sich die Frage, ob der derzeit vielfach praktizierte fragend-entwickelnde Frontalunterricht (zur Bedeutung verschiedender Unterrichtsmethoden in der kaufmännischen Erstausbildung siehe Pätzold, Klusmeyer, Wingels \& Lang 2003, S. $141 \mathrm{ff}$.) diesen Anforderungen gerecht wird. Ein praktisch erprobter und empirisch validierter Ansatz ist das von Sembill entwickelte Didaktikmodell zum Erwerb von Problemlösefähigkeit, "Selbstorganisiertes Lernen" (SoLe, Sembill 1992; Sembill, Schumacher, Wolf, Wuttke \& Santjer-Schnabel 2001). Das zentrale Anliegen des Selbstorganisierten Lernens besteht in der Verbesserung der aligemeinen und domänenspezifischen Fahigkeit von Personen, komplexe ("echte", nicht wohl-definierte) Probleme zu lösen. Damit einher gehen die Ziele Wissensaufbau, Projektplanung, Arbeiten und Lernen in Gruppen, Entwicklung von Selbstbeurteilungsfähigkeit und Präsentationskompetenz u. v. a. mehr. Für den Rechnungswesenunterricht gewendet bedeutet dies, dass Lernende über die Vermittlung der Buchungstechnik hinaus zu ökonomischem Denken zu befähigen sind.

Obwohl Selbstorganisiertes Lernen in mehrfacher Hinsicht Vorteile gegenüber der traditionellen Unterrichtsgestaltung aufweist (Sembill, Wolf, Schumacher \& Wuttke 2002), ist die Verbreitung der Konzeption in der schulischen Praxis mit vielerlei Hindernissen verbunden:

(1) Von Unterrichtspraktikern wird zunächst der hohe Planungs- und Entwicklungsaufwand gesehen. Selbstorganisiertes Lernen ist sicherlich kein "didaktischer Selbstläufer". Die Einführung des Selbstorganisierten Lernens erforderte von den beteiligten Lehrpersonen, gewohnte Unterrichtsmuster zu überdenken, sich mit bis dato nicht geläufigen allgemein- und fachdidaktischen Konzepti-

Konstanzer Online-Publikations-System (KOPS)

URL: http://www.ub.uni-konstanz.de/kops/volltexte/2008/7108/

URN: http://nbn-resolving.de/urn:nbn:de:bsz:352-opus-71085 
onen auseinander $z u$ setzen und umfangreiche Unterrichtsmaterialien ( $z$. B. Problemstellungen und Belegsätze) zu erstellen. Hier bietet es sich an, Unterricht im Team vorzubereiten. ${ }^{1}$ Im Zuge der gemeinsamen Vorbereitung der Lehr-Lern-Sequenzen wird der Unterricht quasi zum kollektiven Eigentum. Das in Lehrerkollegien üblicherweise zu beobachtende Autonomie-Paritäts-Muster kann so ein Stück weit aufgebrochen werden (siehe hierzu Altrichter \& Eder 2004).

(2) Studierende (und auch Lehrkräfte) erhalten während ihrer Aus- und Weiterbildung nicht in ausreichendem Maße Gelegenheit, komplexe Lehr-LernArrangements zu konzipieren. Es ist daher nicht erstaunlich, dass bei vielen Lehrkräften ein hohes Maß an Unsicherheit besteht, wenn es darum geht, komplexere Formen der Unterrichtsgestaltung zu erproben. ${ }^{2}$ Nicht selten fehlt es schlicht und ergreifend an der notwendigen Methodenkompetenz (Pătzold, Klusmeyer, Wingels \& Lang 2003; Klusmeyer \& Pätzold 2005). Hier ist die Lehrerausbildung und Lehrerweiterbildung aufgefordert, bestehende Kompetenzdefizite abzubauen.

Ziel der Bamberger Handelslehrerausbildung ist es, Studierende mit komplexen Lehr-Lern-Arrangements und insbesondere der Konzeption des Selbstorganisierten Lernens vertraut zu machen. Durch entsprechende Präsenzveranstaltung soll sichergestellt werden, dass Studierende Kompetenzen entwicklen, die sie in die Lage versetzen, in der Unterrichtspraxis - auch in Teamarbeit - komplexe Unterrichtsmaterialien zu erstellen, die die Eigenaktivität von Lernenden zielgerichtet unterstützen.

Im Hinblick auf eine erwünschte überregionale Breitenwirkung bietet der Rahmen der virtuellen hochschule bayern (vhb) die Möglichkeit, mittels Online-Seminare auch Studierende an anderen Wirtschaftspädagogikstandorten anzusprechen. Das hier beschriebene Projekt stellt für Studierende, aber auch für Referendare sowie für erfahrene Unterrichtspraktiker ein virtuelles Lehr-Lern-Angebot zur Verfügung, in der die Planung und Konzeption von selbstorganisationsoffenem Unterricht thematisiert und erprobt werden. Die Veranstaltung wird seit dem Wintersemester $2004 / 05$ als so

Bel einer Befragung von knapp 100 Lehrkräften an beruflichen Schulen in Bamberg und Selb (Oberiranken) zum Thema Koperation zwischen Lehrpersonen" rangierte Zeitmangel" an Platz eins der genannten Gründe, die aus Sicht der Befragten gegen eine Kooperation bel der Vorbereeins der genannten Grunde, die aus Sicht der Befr

2 "Gestaltung und Methoden des Unterrichts" ist eine zentrale Kompetenz von Lehrpersonen. Wie "beispielsweise eine Untersuchung von Oser (1997a, 1997b) zeigt, bestehen bei Lehramts Studierenden diesbezüglich erhebliche Defizite. genannter A-Kurs' der vhb angeboten. Da es bisher kein vergleichbares virtuelles Seminar für Wirtschaftspädagogik gibt und das Projekt auch für andere Fachdidaktikbereiche im Rahmen der universitären Lehrerbildung einen exemplarischen Charakter haben könnte, legen wir den Schwerpunkt auf eine detaillierte Darstellung der Gestaltung. Die Ergebnisse einer Evaluation des Seminars werden dagegen nur skizziert. Eine umfassendere Analyse erfolgt zu einem späteren Zeitpunkt.

\section{Konzeption des Online-Seminars}

Virtuelle Lehrveranstaltungen erfordern einen spezifischen Methodeneinsatz, eine ansprechende Aufbereitung und Gestaltung der Lerninhalte, ein detailliertes Betreuungskonzept sowie eine zuverlässige und einfach zu bedienende technische Infrastruktur, mit der die didaktische Gesamtkonzeption auch tatsächlich umgesetz werden können (siehe auch Schulmeister 2001). Diese sollen im folgenden dargestellt werden.

\subsection{Inhaltliche Konzeption}

Die grundlegende Idee des Seminars ist, dass Studierende anhand einer konkreten Problemstellung die Planung und Vorbereitung sowie die Beurteilung von selbstorganisationsoffenem Unterricht praktisch erfahren und theoretisch reflektieren. Aus dem Kanon der kaufmännischen Lernfelder und Themengebiete erscheint insbesondere der tradierte Rechnungswesenunterricht reformbedürftig (Preiß \& Tramm 1996; Preiß 1999; Seifried 2004). So entsteht doppelter Nutzen: Zum einen wird mit der Konzeption "Selbstorganisiertes Lernen" ein wichtiges "Methodenmodul" in die Lehrerausbildung implementiert, zum anderen wird mit dem Rechnungswesen ein zentrales (und bisher reformresistentes) curriculares Kernelement der kaufmännischen Ausbildung zum Gegenstand der Reflektion. Erprobt werden können die entwickelten Unterrichtsmaterialien - zumindest gilt dies für Bamberger Studierende - im paralie dazu angebotenen Seminar "Schulpraktische Übungen".

Dazu wurde das virtuelle Seminar in vier Aufgabenbereiche (Problemstellungen = PS) gegliedert, die von den Teilnehmern bearbeitet werden:

A-Kurse der vhb werden (1) regeimäßig angeboten, (2) tutoriell betreut und (3) durch den Fachrat
der vhb zertifiziert. Entsprechende Kurse sind prüfungsordnungskonform und ermöglichen den Erwerb von Leistungspunkten. 
- Unterricht neu gestalten - moderne Lehr-Lern-Arrangements (PS 1)

- Innovationsfeld kaufmännischer Unterricht - Kritik am ReWe-Unterricht \& Fallbeispiele Selbstorganisationsoffenen Unterrichts (PS 2)

- Unterricht vorbereiten - Problemstellungen und Unterrichtsmaterialien entwickeln (PS 3)

- Lernprozesse beurteilen - alternative Beurteilungsverfahren (PS 4)

Ausgehend von eigenen Erfahrungen mit kaufmännischem Unterricht sollen die Teilnehmerinnen und Teilnehmer die Qualität von Unterricht kritisch reflektieren. Anhand eines frei zu wählenden didaktischen Konzepts werden Vor- und Nachteile gängiger Unterrichtspraktiken herausgestellt und Handlungsempfehlungen zu deren Verbesserung abgeleitet (Problemsteliung 1). Die Lernenden können dabei auf eine umfangreiche Wissensbasis zu innovativen Unterrichtskonzeptionen innerhalb des Kursmaterials zugreifen.

Anschließend setzen sie sich mit fachdidaktischen Fragen des kaufmännischen Unterrichts auseinander. Basierend auf einer theoretischen Reflektion der aktuellen Debatte zum Buchführungsunterricht sollen zunächst Kritikpunkte an der aktuellen Unterrichtspraxis im Bereich Kosten- und Leistungsrechnung gesammelt und diskutiert werden. Danach wird das Konzept des Selbstorganisierten Lernens (SoLe) erarbeitet. In Kleingruppen wird in einem dritten Schritt eine grobe Unterrichtsplanung für eîn Unterrichtshalbjahr in Kosten- und Leistungsrechnung erstellt (Problemstellung 2). Auch hier stehen wieder umfangreiche vertiefende Materialien zur Kritik am Rechnungswesenunterricht, zum Themenbereich Kosten- und Leistungsrechnung und der Konzeption des Selbstorganisierten Lernens zur Verfügung.

Nachdem die Teilnehmerinnen und Teilnehmer ein relativ abstraktes Raster für eine mehrwöchige Unterrichtssequenz erstellt haben, liegt der Fokus anschließend auf der Ausgestaltung einer konkreten Problemstellung ${ }^{1}$ und der dafür benotigten Unterrichtsmaterialien (Problemstellung 3). Ziel ist die detaillierte Planung einer ca. achtstündigen Unterrichtssequenz, in der abschließenden Problemstellung setzen sich die Teilnehmer mit der Notwendigkeit neuer Beurteilungsformen im (SoLe-)Unterricht auseinander. Dem Prinzip der Lerner-Orientierung folgend, beurteilen die Teilnehmerinnen und Teilnehmer im Rahmen der Problemstellung 4 sowohl ihre eigene Lei-

1 Hier sind mit "Problemstellungen" nicht die Abschnitte des Online-Seminars gemeint, sondern die von den Studierenden in PS 2 vorbereiteten Problemstellungen, mit denen sie die Lernenden im Rahmen ihres zu konzipierenden Unterrichts konffontieren möchten. stung in der vorangegangenen Problemsteliung als auch die Leistung der anderen Gruppen.

\subsection{Methodische Gestaltung}

Um die Konzeption des Selbstorganisierten Lernens (SoLe) für die Teilnehmer möglichst anschaulich zu machen, werden die methodische Grundprinzipien von SoLe im virtuellen Seminar seibst umgesetzt. Selbstorganisiertes Lernen dient dabei als Ziel, Inhalt und Methode des Online-Seminars. Dazu werden die in neun Merkmalsbereichen zusammengefassten Bedingungsfaktoren umgesetzt ( $z u$ den Details der neun Merkmalsbereiche siehe Sembill 1992; Wolf 2003). Im Mittelpunkt steht das kollaborative Lösen komplexer, nicht wohl-definierter Probleme. Neben der Zielbildung und der Beurteilung wirken die Teilnehmer maßgeblich an der inhaltlichen Er-

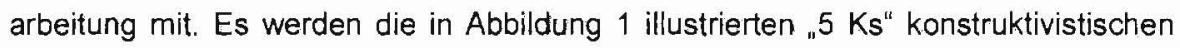
Lernens in Gemeinschaften gefordert und gefördert (Wolf 1995). Damit ergeben sich weitere Lernziele des Seminars auf dem Gebiet der so genannten Schlüsselqualifikationen wie Handlungskompetenz, Projektmanagement, Problemlöse-, Kommunikations- und Kooperationsfähigkeit. Diese oft auch als Soft Skills betitelten Konstrukte stellen zentrale Ziele Selbstorganisierten Lernens dar. Von besonderer Bedeutung ist jedoch die Fähigkeit, komplexe Probleme lösen zu können (Sembill, Schumacher, Wolf, Wuttke \& Santjer-Schnabel 2001).

Abb. 1: Schlüsseltätigkeiten konstruktivistischen Lernens in Gemeinschaften (5 Ks) (Wolf 2003, S. 145)

\begin{tabular}{|c|c|}
\hline Kreation & $\Rightarrow \Rightarrow$ \\
\hline Konstruktion & 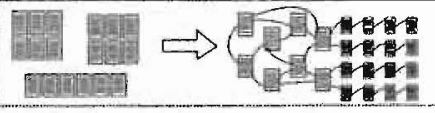 \\
\hline Kommunikation & Smen \\
\hline Kooperation & 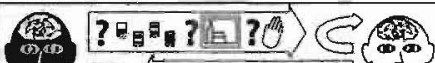 \\
\hline Kollaboration & \\
\hline
\end{tabular}


$\mathrm{Da}$ in einer virtuellen Lernumgebung der unmittelbare Kontakt zum Lehrenden fehlt, bedarf die Durchführung einer virtuellen Lehr-Lern-Verantaltung einer im Vergleich zu einer Präsenzveranstaltung etwas strikteren Lenkung. Deshalb erfolgte die Bearbeitung der vier Problemstellungen jeweils in sechs Phasen (siehe Abb. 2). Die hier gewählte Sechs-Stufen-Systematik kombiniert das Prinzip der vollständigen Handlung (Koch \& Selka 1991, S. 18) mit dem von Roger Schank (Cognitive Arts) für das Online-Lernangebot der Columbia University entwickelten Schemata (siehe Wolf, Städtler \& Baumann 2002)

\section{Abb. 2: Sechsstufiges Durchlaufen jeder einzelnen Problemstellungen}

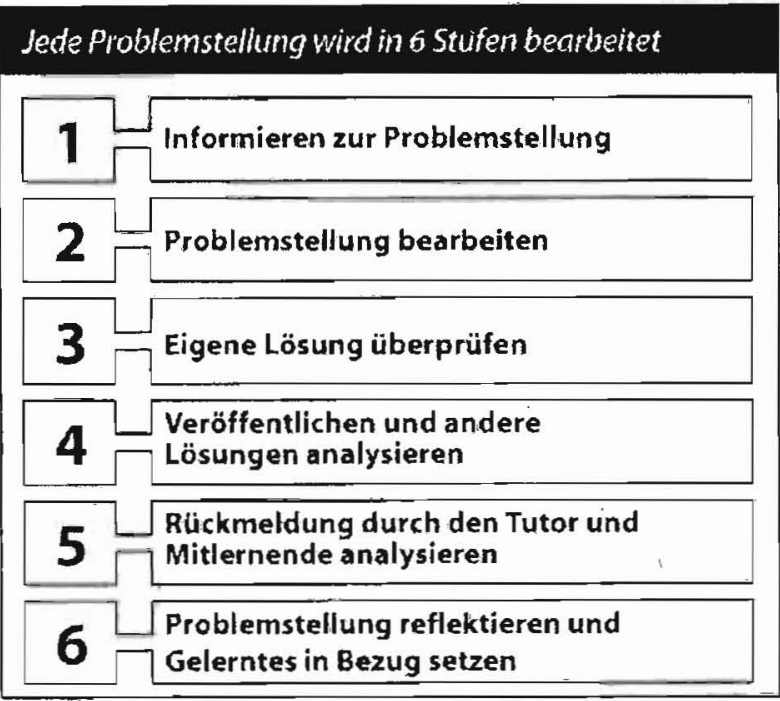

Zur Verstetigung des Seminars ${ }^{1}$ wurden über das SoLe-Konzept hinaus Elemente des Community-of-Practice-Ansatzes nach Wenger (1998) in die Gestaltung des Online-Seminars eingebunden (siehe auch Ausblick). Eine Community of Practice (CoP) ist eine Gruppe, deren Mitglieder eine Leidenschaft oder ein Problem in einem bestimmten Bereich teilen und die ihr Wissen und ihre Expertise durch fortwährende Interaktion auf dem Interessensgebiet vertiefen. Sie teilen Informationen, Ansichten, Ratschläge und helfen einander beim Lösen von domänenspezifischen Problemen
(Wenger, McDermott \& Snyder 2002, S. 4 f.). Die Community of Practice des virtuellen Seminares konstituiert sich über die folgenden Elemente:

- eine Wissensdomane (domain of knowledge): theoretische Fundierung, Vorbereitung, Durchführung und Beurteilung komplexer Lehr-Lern-Arrangements im kaufmännischen Bereich anhand des SoLe-Konzepts;

- eine Gemeinschaft (community) von Mitgliedern, die sich mit der Wissensdomăne beschaftigen: Studenten, Referendare, Lehrende und Forscher;

- eine gemeinsame Praxis (practice), um ihre Effektivität und Effizienz in der Domäne zu steigern: Planung und Reflektion kaufmännischen Unterrichts.

Das CoP-Modell ist keineswegs als intentionales Lehr-Lern-Arrangement Im engeren Sinn zu sehen, sondern es stellt nach Wenger ein Jahrhunderte altes Prinzip sozialen Lernens dar. Es dient in diesem Projekt zur Ergänzung des SoLe-Konzeptes, um die fehlende Präsenzlehre mit ihrer inhärenten Gemeinschaftsbildung zu kompensieren (siehe Rausch 2004)

\subsection{Medientechnische Umsetzung}

Aus den Erfahrungen zur Durchführung internet-unterstützter Lehrveranstaltungen (Wolf 2003) wurden folgende medientechnische und -didaktische Anforderungen definiert:

- Die Online-Arbeit ist durch Präsenzphasen zu ergänzen ("blended learning").

- Die Verständlichkeit der Bedienung (Usability) ist fortlaufend zu evaluieren und bei Bedarf rekursiv im Prozess zu verbessern.

- Die Nutzung des Internets dient nicht primär der Virtualisierung, sondern vor allem der Unterstützung von Kommunikation, Kooperation und Kollaboration.

Zur Umsetzung dieser Anforderungen wurde der Kurs in der Lernumgebung EverLearn (http://www.everlearn.info) implementiert (siehe Abbildung 3) ${ }^{1}$. Neben der Unterstützung der Anbieter von E-Learning-Modulen bei der Erstelłung multimedialer Lerninhalte stellt dieses System zahtreiche Dienste zur Kommunikation (z. B. Instant Messaging, Gruppenchats), zur Kooperation (Diskussionsforen, inhaltsbezogene Rückmeldungen) sowie zur Kollaboration (Bearbeitung gemeinsamer Seiten) bereit.

\footnotetext{
Eine Demoversion findet sich unter hitp://everlearn.bamberg. uni-eriangen.de/demo/sole/

Erfahrungen aus der Implementation neuer Lehr-Lernansatze zeigen, dass „one-shot-training" nicht
funktioniert. Erfolgversprechender ist der andauernde Dialog mit Lehrenden, die kontinuierliche Unterstutzung sowie Reflektion van Herausforderungen, vor denen Lehrpersonen bei de Implementation neuer Programme in den Unterricht stehen (CTGV 1997; Reinmann-Rothmeier \& Mandl 1998) 
In den weiteren Auswertungsschritten werden insbesondere die bestimmenden Faktoren etwaiger Unterschiede im Lernerfolg (Qualităt der Problemlösungen, Ergebnisse der Problemlösefähigkeitstests) sowie das Ausmaß der wahrgenommenen Unterstützung einer Community of Practice analysiert werden.

\section{$4 \quad$ Ausblick}

Die Durchführung der Pilot-Veranstaltung kann vor dem Hintergrund der bisherigen Auswertungen als Erfolg gewertet werden. Die äußerst geringe Drop-Out-Quote (lediglich eine Person brach das Seminar vorzeitig ab) sollte sicherlich nicht überbewertet werden, da es sich bei der Veranstaltung für die Teilnehmer um ein Pflichtseminar handelt, zeigt jedoch auch, dass die Veranstaltungsform als Alternative zu einer traditionellen Präsenzveranstaltung angenommen wird und die glockenförmigen Workload-Verteilung im Seminar angenommen wird. Für die zukünftige Durchführung des Seminars werden wir - auch auf Rückmeldung durch die Seminarteilnehmer - folgende Verbesserungen umsetzen:

- Beispiele für die Erfahrungsberichte und Unterrichtsplanungen anbieten,

- stärkere Nutzung des Gruppenchats zur Beschleunigung erster Diskussionsphasen,

- Einführung wöchentlicher Chatsessions mit den Tutoren,

- Einbindung der core expert group (Sole-Experten, Lehrpersonen) durch Einladung zum Expertenchat und Dokumentation von Projekten der Experten,

- Konzentration auf ein oder zwel Grobkonzeptionen aus PS 2, mit der alle Seminarteilnehmer dann in PS 3 weiterarbeiten sowie

- Einbindung von Video- und Audiomaterial von SoLe-Unterricht und Vorträgen.

Die wichtigste Erkenntnis ist allerdings, dass den Teilnehmern der Prozesscharakter ihrer Lösungsskizzen verdeutlicht werden muss. So wurden z. B. die Unterrichtsplanungen zumeist offline angefertigt und erst anschließend auf den einzelnen Gruppenseiten veröffentlicht. Dies machte aber eine prozessbegleitende Betreuung von Tutoren und Mitlernenden im Sinne von Feedbacks kaum möglich. Die Lernenden betrachteten das Veröffentlichen auf der Gruppenseite als gleichbedeutend mit der endgültigen Abgabe ihrer Arbeit, obwohl die Phasenstruktur der einzelnen Problemstellung im Gegenteil die Veröffentlichung von Zwischenschritten vorsah. Dies ist verständlich, bricht doch diese Vorgehensweise mit der traditionellen Vorgehensweise in Schule und Universität und muss in weiteren Durchläufen bereits in der einführenden Präsenzveranstaltung thematisiert werden.
Inwieweit das Seminar zum Aufbau einer Community of Practice beitragen kann, wird Gegenstand weiterer Untersuchungen sein. Mitglieder dieser Gemeinschaft sollen folgende Personengruppen sein:

- Studierende,

- Mitglieder des Lehrstuhl-Teams Wirtschaftspädagogik,

- Referendare und Lehrer aus dem Fachbereich Wirtschaft,

- externe Experten (Professoren, Dozenten aus der betrieblichen Fort- und Weiterbildung) sowie

- Tutoren.

Für die Umsetzung einer Community ist die Kontinuität der Gemeinschaft unabdingbar. Für die Softwaregestaltung stellt sich vor allem die Frage, wie der Zugang zur Seminarumgebung und den erstellten Materialien auch nach Ablauf der aktiven Seminarphase sinnvoll aufrechterhalten werden kann. Organisatorisch ist zu überlegen wie bei nachfolgenden Seminaren die "Alumnis" den aktuellen Teilnehmerinnen und Teilnehmern durch Feedback hilfreich zur Seite stehen und im Gegenzug auf das dort entwickelte Material zugreifen können. Es solite durch solch einen Austausch gelingen, einen Teil der ehemaligen Seminarteilnehmerinnen und -teilnehmer auch nach Eintritt in das Referendariat an die Gemeinschaft zu binden. Durch den Dialog zwischen Theorie und Praxis und den fortwährend anwachsenden Bestand an einsatzfähigen Unterrichtsmaterialien könnten wir so Lehrpersonen im Vorbereitungsdienst sowie Berufsanfängern eine Plattform für den Aufbau einer langfristigen Community of Practice zur Unterstützung lebenslangen Lernens zur Verfügung stellen. Dies ist der Fokus eines geplanten Forschungsprojektes zur Bildung von Community of Practices in der Lehrerausbildung und Lehrerweiterbildung.

\section{Literatur}

Adl-Amini, B. (1986). Ebenen didaktischer Theoriebildung. In Lenzen, D. (Hrsg.): Enzyklopädie Erziehungswissenschaft. Band 3: Ziele und Inhalte der Erziehung und des Unterrichts. Stuttgart: Klett-Cotta, S. 27-48.

Altrichter, H. \& Eder, F. (2004): Das „Autonomie-Paritäts-Muster" als Innovationsbarriere?, in Holtappels, G. (Hrsg.): Schulprogramme - Instrument der Schulentwicklung. Konzeptionen, Forschungsergebnisse. Praxisempfehlungen. Weinheim und München: Juventa, $S$ nen, Forschi
$195-221$.

Brugia, M. (2004): E-learning for teachers and trainers. Innovative practices, skills and competences. Thessaloniki: Cedefop. 
Zunächst fallen die durchgängig günstigeren Werte der SoLe-Klassen gegenüber den Kontrollklassen mit traditionellem Unterricht (TraLe II / Trale III) auf. Im Vergleich zu den Benchmarkstudien SoLe II und SoLe III sind die Motivationswerte ăhnlich hoch. Während die Werte für die Kompetenzunterstützung und Autonomie erwartungsgemäß hoch sind, ergeben sich im Vergleich zu den Ergebnissen von Wolf (2003) in einem ähnlichen Setting (teil-virtuelles Seminar im Universitätskontext) jedoch einige interessante Unterschiede. So sind die Werte für die soziale Einbindung sowie die Instruktionsqualitat höher. Hier gilt es zu untersuchen, ob im regulären $\mathrm{Be}$ trieb die starke soziale Einbindung auch bei einer größeren regionalen Streuung der Teilnehmer gehalten werden kann. Für die hohe wahrgenommene Instruktionsqualităt scheinen zwei Faktoren ausschlaggebend zu sein: die deutliche Strukturierung der - weiterhin komplexen - Problemstellungen sowie die deutlich intensivere tutorielle Betreuung. Insgesamt zeigt die Gegenüberstellung der internetgestützen SoLeSettings (SoLe vhb und EduSerf) im Vergleich zu den anderen SoLe-Untersuchungen eine hohe Eignung dieser Lehr-Lern-Konzeption für virtuelle Lehr-LernArrangements.

Im Bezug auf den Selbstorganisationsgrad des Seminars sollen hier die drei wichtigsten Abweichungen zu bisherigen SoLe-Studien berichtet werden (siehe Tabelle 2; siehe zur Diskussion des Fragebogens Selbstorganisationsgrad von Unterricht Wolf 2003, S. 245 ff. und Seifried 2004, S. 184 ff.). Durch den hohen Anwendungscharakter konnte die Praxisrelevanz weiter gesteigert werden. Inwieweit dadurch ggf. die Qualität der theoretischen Reflektion gelitten hat, konnen erst weitere Detailanalysen der Tests zur Problemlösefähigkeit verraten. Die Komplexität der Problemstellungen spiegelt sich wider in der starken Verneinung des Items "Es gab immer nur eine richtige Lösung für ein Problem". Hier wurde also fach-inhaltlich die Faktorenkomplexion (Adl-Amini 1986) der Unterrichtsvorbereitung adaquat abgebildet. Ein Resultat der expliziten Gestaltung eines Tutorenleitfadens und konstruktiven Fehlermanagements nach Frese (1995) und dessen erfolgreicher Umsetzung ist die äußerst hohe Zustimmung zum Item "Fehier als Chance".
Tab. 1: Ergebnisse Motivationsfragebogen nach Prenzel (Skalenmittelwerte, die zwei günstigsten Werte einer Skala sind fett, die zwei ungünstigsten kursiv gedruckt) ${ }^{\uparrow}$

\begin{tabular}{|c|c|c|c|c|c|c|c|}
\hline \multirow[b]{2}{*}{ Item } & \multirow[b]{2}{*}{ Sole vhb } & \multirow[b]{2}{*}{ EduSerf } & \multirow[b]{2}{*}{ SoLe II } & \multicolumn{2}{|c|}{ SoLe III } & \multicolumn{2}{|c|}{ TraLe } \\
\hline & & & & EG1 & EG2 & II & III \\
\hline Amotivation & 1,82 & 2,17 & 2,31 & 2,00 & 2,19 & 2,47 & 2,53 \\
\hline Extrinsische Motivation & 2,21 & 1,93 & 2,45 & 2,22 & 2,52 & 2,58 & 2.55 \\
\hline Introjizierte Molivation & 4,86 & 3,90 & 4,07 & 4,33 & 4,27 & 4,20 & 4,14 \\
\hline Identifizierte Motivation & 4,75 & 4,73 & 4,68 & 4,80 & 4,53 & 4,02 & 4.51 \\
\hline Intrinsische Motivation & 4,48 & 4,73 & 4,27 & 4,18 & 4,00 & 3,04 & 3,20 \\
\hline Interesse & 4,11 & 4,80 & 3,76 & 3,92 & 3,51 & 3,24 & 3,04 \\
\hline \multicolumn{8}{|l|}{ Bedingungsfaktoren } \\
\hline Negative Empfindungen & 3,02 & 2,00 & 2,74 & 2,55 & 2,87 & 3,09 & 3,11 \\
\hline Positive Empfindungen & 4,24 & 4,77 & 3,77 & 3,87 & 3,40 & 2,73 & 2,87 \\
\hline Empfundene Wichtigkeit & 4,23 & 4,50 & 4,43 & 4,79 & 4,54 & 3,93 & 4,59 \\
\hline Soziale Einbindung & 4,79 & 4,25 & 4.27 & 4,85 & 4,17 & 3,98 & 3,52 \\
\hline Kompetenzunterstutzung & 4,47 & 4,10 & 4,04 & 4,17 & 3,82 & 3,35 & 3,36 \\
\hline Autonomie & 4,72 & 4,99 & 4,28 & 4.24 & 3,81 & 3.44 & 3,28 \\
\hline Instruktionsqualität & 4,55 & 3,65 & 4,49 & 4,58 & 4,53 & 3,94 & 3,88 \\
\hline $\begin{array}{l}\text { Überforderung vs. Anpas- } \\
\text { sung }\end{array}$ & 3,14 & 2,27 & 3,53 & 3,07 & 3,27 & 3,13 & 3,02 \\
\hline
\end{tabular}

Tab. 2: Vergleich ausgewählter Items des Fragebogens Selbstorganisationsgrad von Unterricht (SoGU) (Skalenmittelwerte, die zwei günstigsten Werte einer Skala sind fett, die zwei ungünstigsten kursiv gedruckt) ${ }^{2}$

\begin{tabular}{|c|c|c|c|c|c|c|c|}
\hline \multirow[b]{2}{*}{ Item } & \multirow[b]{2}{*}{ SoLe vhb } & \multirow[b]{2}{*}{ EduSerf } & \multirow[b]{2}{*}{ SoLe II } & \multicolumn{2}{|c|}{ SoLe III } & \multicolumn{2}{|c|}{ TraLe } \\
\hline & & & & EG1 & EG2 & & III \\
\hline $\begin{array}{l}\text { Was ich im Seminar ge- } \\
\text { lernt habe, kann ich } \\
\text { (später) auch in der Schule } \\
\text { anwenden. }\end{array}$ & 4,88 & 4,67 & 3,87 & 3,81 & 3,36 & 3,73 & 3,85 \\
\hline $\begin{array}{l}\text { Es gab immer nur eine } \\
\text { richtige Lösung für ein } \\
\text { Problem. }\end{array}$ & 1,88 & 1,67 & 3,20 & 3,31 & 3,27 & 3,13 & 4,35 \\
\hline $\begin{array}{l}\text { Im Seminar wurden Fehler } \\
\text { als Chance gesehen, die } \\
\text { Inhalte besser zu verste- } \\
\text { hen. }\end{array}$ & 4,98 & 4,78 & 4,47 & 4,81 & 4,86 & 4,00 & 4,90 \\
\hline
\end{tabular}

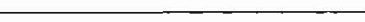

Die Schüler wurden Jeweils gebeten, auf einer sechsstufigen Skala (von $1=$ "nie” bis $6=$ "Immer") zu beurteilen, wie of sie die beschriebenen motivationalen Zustände im Unterricht erreicht haben Für die Subskalen "Amotivation" und "Extrinsische Motlvation" sind niedrigere Werte

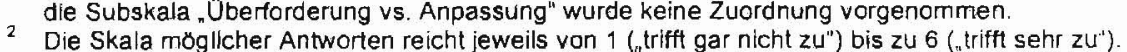


Zusammenfassend lasst sich zur Benotungskonzeption sagen, dass wir hier eine auf Arbeitsergebnisse und -dokumentationen basierende Prozessbeurteilung realisieren, die durch Selbst- und Peer-Beurteilung ergänzt wird.

\subsection{Workload und ECTS-Punkte}

Die Arbeitsbelastung steigt zu Beginn des Seminars langsam an, um eine allmähliche Eingewöhnung zu ermöglichen und somit dem häufig beobachteten „Abspringen" (Drop-Out) der Studierenden bei Online-Lernangeboten entgegenzuwirken. Nach PS 3 nimmt die Beanspruchung wieder ab, da die Studentinnen und Studenten in dieser Zeit erfahrungsgemäß mit der Vorbereitung auf anstehende Klausuren beginnen.

Der Workload im Seminar liegt gemäß einer Anrechnung von 3 ECTS-Punkten bei insgesamt circa 90 Stunden. Das Modul umfasst 4 SWS (2 Std. Seminar + 2 Std. Übung) zzgl. Vor- und Nachbereitung. Aufgrund der zeitlichen Stauchung der aktiven Seminarphase von 15 auf 12 Semesterwochen ergibt sich ein durchschnittlicher Arbeitsaufwand von circa acht Stunden pro Woche, der allerdings über das Semester hinweg nicht gleich verteilt ist.

\section{Erste empirische Befunde der Evaluation des Seminars}

Bevor das Seminar im Veranstaltungsprogramm der Virtuellen Hochschule Bayern (vhb) angeboten werden konnte, war zunächst zu klären, ob die didaktischen Anforderungen der vhb erfült werden, ob die Konzeption der Veranstaltung von den Teilnehmern angenommen wird und inwiefern ggf. Änderungsbedarf besteht. Darüber hinaus versuchten wir zu klären, ob die Einbindung des Community of PracticeAnsatzes in Kombination mit Selbstorganisiertem Lernen in einem virtuellen Setting funktioniert. Im Folgenden sollen einige erste Ergebnisse der Evaluation insbesondere bezüglich der Einschätzung des Seminars durch die Teilnehmer berichtet werden.

Das Seminar wurde im Sommersemester 2004 als Pilotveranstaltung am Lehrstuh für Wirtschaftspädagogik in Bamberg durchgeführt. Es nahmen 46 Studentinnen und Studenten der Otto-Friedrich-Universităt Bamberg teil, die sich zum überwiegenden Teil im vierten Fachsemester des Studiengangs Wirtschaftspädagogik bzw. Wirtschaftspädagogik mit dem Schwerpunkt Informationstechnologie (Wirtschaftspädagogik/lT) befanden. Eine Person brach das Seminar nach zwei Wochen ab ( out"). Für die weitere Auswertung wurden zwei weitere Teilnehmer aufgrund entschuldigter Fehizeiten aus dem Datensatz herausgenommen. Die untersuchte Seminargruppe setzte sich aus 20 Männern und 23 Frauen zusammen $(n=43)$. Das Erhebungsdesign sah neben einer Eingangserhebung und einer Ausgangserhebung die wiederholte Zwischenerhebung eines "Community of Practice"-Barometers zur Messung der wahrgenommenen Gemeinschaftsbildung vor (zu den Details der eingesetzten Instrumente siehe Rausch 2004).

Da die Erstellung eigener Inhalte innerhalb der Lernumgebung unverzichtbarer Bestandteil der Seminarkonzeption war, fragten wir mit dem Fragebogen Computervorwissen und Interesse an Computer und Intemet (CoVI, Wolf 2004) den Kenntnisstand der Teilnehmer ab. $90 \%$ der Teilnehmer hatten bisher keine eigene Homepage im Internet, ca. $75 \%$ hatten bisher noch nie einen HTML-Editor zur Gestaltung von Webseiten benutzt. Im Rahmen der ersten Präsenzveranstaltung gelang es ungeachtet der geringen Vorkenntnisse der Teilnehmerinnen und Teilnehmer - die Studierenden mit der Erstellung von Inhalten in EverLearn vertraut zu machen. Alle Lernenden hatten nach 30 Minuten eine eigene Homepage gestaltet. Über den Verlauf der aktiven Seminarphase (81 Tage) loggten sich die Studierenden durchschnittlich alle 2,15 Tage in das System ein, was als außerst regelmäßige und aktive Teilnahme interpretiert werden kann.

Im Folgenden konzentrieren wir uns auf die Ergebnisse aus der Ausgangserhebung. Zur Erfassung der Motivation der Lernenden wurde ein von der Forschergruppe um Prenzel (siehe z. B. Prenzel, Kristen, Dengler, Ettle \& Beer 1996) entwickelter Fragebogen zur Lernmotivation adaptiert und eingesetzt. Die Ergebnisse des Motivationsfragebogens nach Prenzel gibt Tabelle 1 wieder. Zur besseren Einordnung der Ergebnisse werden die Ergebnisse aus dem Online-Seminar den Befunden aus insgesamt drei Benchmark-Studien gegenübergestellt:

- SoLe II (DFG-Schwerpunktprogramm "Lehr-Lern-Prozesse in der kaufmännischen Erstausbildung", $n=15$, Bürokaufleute, Lerninhalt "Personalwirtschaft", siehe Sembill 1999, 2000, 2004; Sembill, Schumacher, Wolf, Wuttke \& SantjerSchnabel 2001; Klüber 2003),

- SoLe III mit zwei Experimentalgruppen ( $n=44$, kaufmännische Grundstufe, Lerninhalt "Buchführung", siehe hierzu Seifried 2004, Seifried \& Sembill 2004 sowie Seifried, Wolf, Klüber \& Sembill in diesem Band) sowie

- SoLe-EduSerf $(n=11$, teilvirtuelles Seminar, Wolf 2003) 
Zunächst fallen die durchgängig günstigeren Werte der SoLe-Klassen gegenüber den Kontrollklassen mit traditionellem Unterricht (TraLe II / Trale III) auf. Im Vergleich zu den Benchmarkstudien SoLe II und SoLe III sind die Motivationswerte ăhnlich hoch. Während die Werte für die Kompetenzunterstützung und Autonomie erwartungsgemäß hoch sind, ergeben sich im Vergleich zu den Ergebnissen von Wolf (2003) in einem ähnlichen Setting (teil-virtuelles Seminar, im Universitătskontext) jedoch einige interessante Unterschiede. So sind die Werte für die soziale Einbindung sowie die Instruktionsqualităt höher. Hier gilt es zu untersuchen, ob im regulären Betrieb die starke soziale Einbindung auch bei einer größeren regionalen Streuung der Teilnehmer gehalten werden kann. Für die hohe wahrgenommene Instruktionsqualität scheinen zwei Faktoren ausschlaggebend zu sein: die deutliche Strukturierung der - weiterhin komplexen - Problemstellungen sowie die deutlich intensivere tutorielle Betreuung. Insgesamt zeigt die Gegenüberstellung der internetgestützen SoLeSettings (SoLe vhb und EduSerf) im Vergleich zu den anderen SoLe-Untersuchungen eine hohe Eignung dieser Lehr-Lern-Konzeption für virtuelle Lehr-LernArrangements.

Im Bezug auf den Selbstorganisationsgrad des Seminars sollen hier die drei wichtigsten Abweichungen zu bisherigen SoLe-Studien berichtet werden (siehe Tabelle 2; siehe zur Diskussion des Fragebogens Selbstorganisationsgrad von Unterricht Wolf 2003, S. 245 ff. und Seifried 2004, S. 184 ff.). Durch den hohen Anwendungscharakter konnte die Praxisrelevanz weiter gesteigert werden. Inwieweit dadurch ggf. die Qualität der theoretischen Reflektion gelitten hat, können erst weitere Detailanalysen der Tests zur Problemlösefähigkeit verraten. Die Komplexität der Problemstellungen spiegelt sich wider in der starken Verneinung des Items ${ }_{n}$ Es gab immer nur eine richtige Lösung für ein Problem". Hier wurde also fach-inhalttich die Faktorenkomplexion (Adi-Amini 1986) der Unterrichtsvorbereitung adaquat abgebildet. Ein Resultat der expliziten Gestaltung eines Tutorenleitfadens und konstruktiven Fehlermanagements nach Frese (1995) und dessen erfolgreicher Umsetzung ist die äußerst hohe Zustimmung zum Item "Fehier als Chance".
Tab. 1: Ergebnisse Motivationsfragebogen nach Prenzel (Skalenmittelwerte, die zwei günstigsten Werte einer Skala sind fett, die zwei ungünstigsten kursiv gedruckt)

\begin{tabular}{|c|c|c|c|c|c|c|c|}
\hline \multirow[b]{2}{*}{ Item } & \multirow[b]{2}{*}{ Sole vhb } & \multirow[b]{2}{*}{ EduSerf } & \multirow[b]{2}{*}{ SoLe II } & \multicolumn{2}{|c|}{ SoLe III } & \multicolumn{2}{|c|}{ TraLe } \\
\hline & & & & EG1 & EG2 & ॥ & III \\
\hline Amotivation & 1,82 & 2,17 & 2,31 & 2,00 & 2,19 & 2,47 & 2,53 \\
\hline Extrinsische Motivation & 2,21 & 1,93 & 2,45 & 2,22 & 2,52 & 2,58 & 2.55 \\
\hline Introjizierte Molivation & 4,86 & 3,90 & 4,07 & 4,33 & 4,27 & 4,20 & 4,14 \\
\hline Identilizierte Motivation & 4,75 & 4,73 & 4,68 & 4,80 & 4,53 & 4,02 & 4.51 \\
\hline Intrinsische Motvation & 4,48 & 4,73 & 4,27 & 4,18 & 4,00 & 3.04 & 3,20 \\
\hline Interesse & 4,11 & 4,80 & 3,76 & 3,92 & 3,51 & 3,24 & 3,04 \\
\hline \multicolumn{8}{|l|}{ Bedingungsfaktoren } \\
\hline Negative Empfindungen & 3,02 & 2,00 & 2,74 & 2,55 & 2,87 & 3,09 & 3,11 \\
\hline Positive Empfindungen & 4,24 & 4,77 & 3,77 & 3,87 & 3,40 & 2,73 & 2,87 \\
\hline Empfundene Wichtigkeit & 4,23 & 4,50 & 4,43 & 4,79 & 4,54 & 3.93 & 4,59 \\
\hline Soziale Einbindung & 4,79 & 4,25 & 4.27 & 4,85 & 4,17 & 3.98 & 3,52 \\
\hline Kompetenzunterstutzung & 4,47 & 4,10 & 4,04 & 4,17 & 3,82 & 3,35 & 3,36 \\
\hline Autonomie & 4,72 & 4,99 & 4,28 & 4,24 & 3,81 & 3.44 & 3,28 \\
\hline Instruktionsqualität & 4,55 & 3,65 & 4,49 & 4,58 & 4,53 & 3,94 & 3,88 \\
\hline $\begin{array}{l}\text { Uberforderung vs. Anpas- } \\
\text { sung }\end{array}$ & 3,14 & 2,27 & 3,53 & 3,07 & 3,27 & 3,13 & 3,02 \\
\hline
\end{tabular}

Tab. 2: Vergleich ausgewăhlter Items des Fragebogens Selbstorganisationsgrad von Unterricht (SoGU) (Skalenmittelwerte, die zwei günstigsten Werte einer Skala sind fett, die zwei ungünstigsten kursiv gedruckt) ${ }^{2}$

\begin{tabular}{|c|c|c|c|c|c|c|c|}
\hline \multirow[b]{2}{*}{ Item } & \multirow[b]{2}{*}{ SoLe vitb } & \multirow[b]{2}{*}{ EduSerf } & \multirow[b]{2}{*}{ SoLe II } & \multicolumn{2}{|c|}{ SoLe III } & \multicolumn{2}{|c|}{ TraLe } \\
\hline & & & & EG1 & EG2 & & 圳 \\
\hline $\begin{array}{l}\text { Was ich im Seminar ge- } \\
\text { lernt habe, kann ich } \\
\text { (später) auch in der Schule } \\
\text { anwenden. }\end{array}$ & 4,88 & 4,67 & 3,87 & 3,81 & 3,36 & 3,73 & 3,85 \\
\hline $\begin{array}{l}\text { Es gab immer nur eine } \\
\text { richtige Lösung für ein } \\
\text { Problem. }\end{array}$ & 1,88 & 1,67 & 3,20 & 3,31 & 3,27 & 3,13 & 4,35 \\
\hline $\begin{array}{l}\text { In Seminar wurden Fehler } \\
\text { als Chance gesehen, die } \\
\text { Inhalte besser zu verste- } \\
\text { hen. }\end{array}$ & 4,98 & 4,78 & 4,47 & 4,81 & 4,86 & 4,00 & 4,90 \\
\hline
\end{tabular}

Die Schüler wurden Jeweils gebeten, auf einer sechsslufigen Skala (von $1={ }_{\text { nie" }}$ bis $6=$ "immer") zu beurleilen, wie oft sie die beschiriebenen molivationalen Zustande im Unterricht erreicht haben. Fur die Subskalen "Amolivation" und "Extrinsische Molvation" sind niedrigere Werte günstiger. Fü de Subskala "n 
Abb. 5: Aufgaben der Tutoren in den einzelnen Problemstellungen

\begin{tabular}{|c|c|c|}
\hline \multicolumn{2}{|c|}{ Problemstellung } & Aufgaben der Tutoren \\
\hline PS1: & $\begin{array}{l}\text { Anfertigen eines } \\
\text { kurzen Erfah- } \\
\text { rungsberichts }\end{array}$ & $\begin{array}{l}\text { - Halten die Teilnehmer an, den Erfahrungsbericht im angegebenen Zeit- } \\
\text { rahmen zu erstellen und geben ggf. Hilfestellung zur inhaltichen } \\
\text { Gestaltung. }\end{array}$ \\
\hline PS1: & $\begin{array}{l}\text { Kritische Diskus- } \\
\text { sion anhand einer } \\
\text { ausgewähtten Un- } \\
\text { terrichtsmethode" }\end{array}$ & $\begin{array}{l}\text { - Geben inhaltliches Feedback zu den erstellten Kommentaren der Stu- } \\
\text { dierenden. Dabei wird besonders auf den inhaltlichen Bezug des } \\
\text { Kommentars zu der ausgewählten Unterrichtsmethode geachtet. } \\
\text { - Stellen sich mit ihrem Feedback der Diskussion mit den Studjerenden. } \\
\text { - Versuchen, Diskussionen unter den Teilnehmern anzuregen. } \\
\text { - Bewerten die erstellten Kommentare und erstellen einen Notenvor- } \\
\text { schlag für die Bearbeitung der Problemstellung. }\end{array}$ \\
\hline PS2: & $\begin{array}{l}\text { Sammeln und } \\
\text { Diskutieren von } \\
\text { Kritikpunkten am } \\
\text { KLR-Unterricht }\end{array}$ & $\begin{array}{l}\text { - Stoßen die Diskussion an und versuchen die Studierenden entspre- } \\
\text { chend einzubinden. } \\
\text { - Sichten Kritikpunkte und fassen wichtigste Ergebnisse zusammen. }\end{array}$ \\
\hline PS2: & $\begin{array}{l}\text { Das Konzept des } \\
\text { Selbstorganisier- } \\
\text { ten Lernens } \\
\text { (SoLe) }\end{array}$ & $\begin{array}{l}\text { - Stehen während der Informationsphase für Fragen der Studierenden } \\
\text { zum SoLe-Konzept zur Verfügung. } \\
\text { - Unterstützen die Bildung von Kleingruppen zur Bearbeitung der weite- } \\
\text { ren Aufgaben. }\end{array}$ \\
\hline PS2: & $\begin{array}{l}\text { Planung einer } \\
\text { mehrwöchigen } \\
\text { Unterrichts- } \\
\text { sequenz }\end{array}$ & $\begin{array}{l}\text { - Geben Feedback zu den Gruppenarbeitsergebnissen (Unterrichts- } \\
\text { plänen) gemäß formaler und fachlicher Kriterien. } \\
\text { - Halten die Gruppen zur Selbstbewertung an und erstellen auf Basis } \\
\text { dieser Selbstbewertung und der erstellten Arbeitsergebnisse einen No- } \\
\text { tenvorschlag für die Bearbeitung der Problemstellung. }\end{array}$ \\
\hline PS3: & Feinplanung & $\begin{array}{l}\text { Regen die Gruppen zu wechseiseitigem Feedback an. Die Tutoren ge- } \\
\text { ben - falls notwendig - ein kurzes Feedback zu den in Gruppenarbeit } \\
\text { erstellten Feinplanungen gemäß formaler und fachlicher Kriterien. }\end{array}$ \\
\hline PS3: & $\begin{array}{l}\text { Erstellung von } \\
\text { Unterrichts- } \\
\text { material }\end{array}$ & $\begin{array}{l}\text { - Regen dle Gruppen zu wechselseitigem Feedback an. Die Tutoren ge- } \\
\text { ben, falls notwendig, ein kurzes Feedback zu den in Gruppenarbeit } \\
\text { erstellten Unterrichtsmaterialen gemäß formaler und fachlicher Kriterien } \\
\text { (inbes. Originalität und Realitätsnähe). } \\
\text { - Überprüfen die Ergebnisse des Peer-Reviews der zweiten Präsenzver- } \\
\text { anstaltung und erstellen einen Notenvorschlag für die Bearbeitung der } \\
\text { Problemstellung. }\end{array}$ \\
\hline PS4: & $\begin{array}{l}\text { "Self-Assess- } \\
\text { ment" }\end{array}$ & $\begin{array}{l}\text { - Beurteilen die Selbstbewertung der Teilnehmer gemäß ausgewähiter } \\
\text { Kriterien für die Güte von Bewertungsprozessen. }\end{array}$ \\
\hline PS4: & Gesamtleistung & - Notenvorschlag für die Bearbeitung der Problemstellung 4. \\
\hline & $\begin{array}{l}\text { bis PS4: Gesamt- } \\
\text { note }\end{array}$ & $\begin{array}{l}\text { - Notenvorschlag für dle Gesamtleistung im Seminar auf Basis der in den } \\
\text { vier Problemstelfungen erbrachten Leistungen unter Berücksichtigung } \\
\text { deren spezifischer Gewichtung. }\end{array}$ \\
\hline
\end{tabular}

\footnotetext{
Dabei geben die Tutoren konstruktive Verbesserungsvorschläge und weisen, wenn möglich, auf Ergebrisse anderer Gruppen hin. Auf diese Weise fördern die Tutoren die Diskussion und den Erlahrungsaustausch zwischen den einzelnen Gruppen mif dem Ziel, unterstützende Tätigkeiten im weiteren Seminarablauf zurückzufahren („fading").
}

\subsection{Beurteilungskonzept}

Das Seminar "Selbstorganisationsoffener Unterricht am Beispiel Rechnungswesen" ist ein Pflichtseminar im Hauptstudium des Studiengang Wirtschaftspädagogik an der Universität Bamberg. Die Studierenden an der Universităt Bamberg können zwischen einer Online- und einer Präsenzvariante wăhlen, wobei jedoch letztere nur im Wintersemester angeboten wird. Mit dem Online-Seminar ist also eine flexiblere Gestaltung des Studiums möglich. Für Studierende an anderen bayerischen Hochschulen wird die Chance geschaffen, sich mit diesem Konzept zu beschäftigen.

Da das kollaborative Lösen komplexer Probleme im Mittelpunkt des Selbstorganisierten Lernens steht, greifen traditionelle Beurteilungsformen wie z. B. Klausuren hier zu kurz. Dementsprechend wurde ein adäquates Beurteilungskonzept entwickelt:

- Die Gesamtnote für das Seminar setzt sich aus vier Teilnoten für die vier Problemstellungen zusammen, die - entsprechend der Arbeitsintensitatt - folgendermaßen gewichtet werden: PS 1 und PS 4 je 20\%, PS 2 und PS 3 je $30 \%$.

- Die Bewertung erfolgt durch den verantwortlichen Seminarleiter in Absprache mit den Tutoren.

- Bei kooperativen Problemlösungen wird jeweils die gesamte Gruppenleistung bewertet, d. h. die Gruppenmitglieder werden einheitlich benotet. Für eine angemessene Lastenverteilung innerhalb der Gruppe sind die Lernenden selbst verantwortlich.

- Auch innerhalb der einzelnen Problemstellungen richtet sich die Gewichtung der Teilaufgaben nach der Arbeitsintensität. So wird in PS 1, PS 2 und PS 4 der jeweils letzten Teilaufgabe eine größere Bedeutung beigemessen als der ersten

- Während der Problemsteilungen von den Tutoren gegebene Hinweise, Anregungen und Kritiken haben keinen negativen Einfluss auf die Note. Das Feedback soll als Hilfe, nicht als Strafe verstanden werden.

- Bei der Beteiligung in Diskussionsforen steht die Qualität - nicht die Quantität der Diskussionsbeiträge im Vordergrund.

- Eine Besonderheit ergibt sich für PS 3, da die in PS 4 erfolgten Benotungen (sowohl Eigen- als auch Fremdbeurteilung) ergänzt durch eine Benotung der Tutoren zu je einem Drittel in diese Teilnote eingehen. Die Teilnote ermittelt sich gleichgewichtet aus Selbst-, Peer- und Fremdbeurteilung

- Das geforderte regelmäßige Engagement (mit Phasen relativ hoher Belastung) rechtfertigt, dass kein weiterer Leistungsnachweis (z. B. in Form einer Klausur oder einer Hausarbeit) erbracht werden muss. 
Cognltion and Technology Group at Vanderbilt (1997): The Jasper project: Lessons in curriculum, instruction, assessment, and professional development, Mahwah (NJ): Erlbaum.

Egloffstein, M. (2004): Tutorenleitfaden für die Tutoren des vhb Online Seminars Selbstorganisationsoffener Unterricht am Beispiel Rechnungswesen. Unveröffentlichtes Manuskript.

Frese, M. (1995): Error management in training: Conceptual and empirical results, in Zucchermaglio, C., Bagnara, S. \& S. Stucky, U. (Eds.), Organizational learning and technological change. Berlin, Heideiberg, New York: Springer, pp. 112-124.

Klüber, C. (2003): Wie erleben Schülerinnen und Schüler Unterricht?, in: van Buer,J. \& Zlatkin-Troitschanskaia, O. (Hrsg.): Berufliche Bildung auf dem Prüfstand, Frankfurt a. M. U. a.: Peter Lang, S. 257-284.

Klusmeyer, J. \& Pätzold, G. (2005): Die untertichtsmethodische Kompetenz von Lehrkräften an kaufmännischen Berufsschulen als mögliches Impiementationsproblem des Lernfeldkonzepts, in: Wirtschaft und Erziehung, 57. Jg., H. 1, S. 11-15.

Koch, J., Selka, R. (1991): Leittexte - ein Weg zu selbstandigem Lernen. Veranstalter-Info, Berlin: BIBB, 2. Auflage.

Pätzold, G., Klusmeyer, J., Wingels, J. \& Lang, M. (2003): Lehr-Lern-Methoden in der beruflichen Bildung. Eine empirische Untersuchung in ausgewähiten Berufsfeldern, Oldenburg: Universität Oldenburg.

Oser, F. (1997a): Standards in der Lehrerbildung. Teil 1: Berufliche Kompetenzen, die hohen Qualitätsmerkmalen entsprechen, in: Beiträge zur Lehrerbildung, 15. Jg., H. 1, S. 26-37.

Oser, F. (1997b): Standards in der Lehrerbildung. Teil 2: Wie werden Standards in der schweizerischen Lehrerbildung erworben? Erste empirische Ergebnisse, in: Beiträge zur Lehrerbildung, 15. Jg., H. 2, S. 210-228.

Preiß, P. (1999): Didaktik des wirtschaftsinstrumentellen Rechnungswesens, München \& Wien: Oldenbourg

Preiß, P. \& Tramm, T. (1996): Die Gottinger Unterrichtskonzeption des wirtschaftsinsirumentellen Rechnungswesens, in: Preiß, P. \& Tramm, T. (Hrsg.) Rechnungswesenunterricht und ökonomisches Denken. Didaktische Innovationen für die kaufmännische Ausbildung, Wiesbaden: Gabler, S. 222-323.

Rausch, A. (2004): Wissensaustausch innerhaib eines virtuellen Lehr-Lern-Arrangements Selbstorganisiertes Lernen in "Communilies of Practice". Bamberg: unveröffentlichte Diplomarbeit.

Rautenstrauch, C. (2001): Tele-Tutoren: Qualifizierungsmerkmale einer neu entstehenden Profession. Bielefeld: Bertelsmann.

Reinmann-Rothmeier, G. \& Mandl, H. (1998): Wenn kreative Ansätze versanden. Implementation als verkannte Aufgabe, in: Unterrichtswissenschaft, 26. Jg., H. 4, S. 292-311.

Schuimeister, R. (2001): Virtuelle Universităt - Virtuelles Lernen, München \& Wien: Oldenbourg.
Seifried, J. (2004): Fachdidaktische Variationen in einer selbstorganisationsoffenen Lernumgebung - Eine empirische Untersuchung des Rechnungswesenunterrichts, Wiesbaden Deutscher Universităts-Verlag.

Seifried. J. \& Sauer, C. (im Druck): Gemeinsam statt allein - Kooperation zwischen Lehrpersonen an beruflichen Schulen, in: VLB-Akzente.

Seifried, J. \& Sembill, D. (2004): Rechnungswesenunterricht neu gestalten. Empirische Befunde aus einem Kooperationsprojekt zwischen Schule und Hochschule, in: Wirtschaf und Erziehung, H. 11, 56. Jg. S. 371-378.

Seifried, J., Wolf, K. D., Kluber, C. \& Sembill, D. (2005): Die Kompatibilităt curricularer und methodischer Modellierungen als notwendige Bedingung für Unterrichtsqualität. In: Sembill, D. \& Seifried, J. (Hrsg.): Rechnungswesenunterricht am Scheideweg: Lehren, Lernen und Prüfen. Wiesbaden: Deutscher Universilăts-Verlag, S. 123-142.

Sembill, D. (1992): Problemlösefähigkeit, Handlungskompetenz und Emotionale Befindlichkeit. Zielgrößen Forschenden Lernens, Göttingen, Toronto \& Zürich: Hogrefe.

Sembill, D. (1999): Selbstorganisation als Modellierungs-, Gestaltungs- und Erforschungsidee beruflichen Lernens, in: Tramm, T., Sembill, D., Klauser, F. \& John, E. G (Hrsg.): Professionalisierung kaufmännischer Berufsbildung: Beiträge zur Öfnung de Wirtschaftspädagogik für die Anforderungen des 21. Jahrhunderts. Festschrift zum 60 Geburtstag von Frank Achtenhagen, Frankfurt/Main u.a.: Lang, S. 146-174.

Sembill, D. (2000): Selbstorganisiertes und Lebenslanges Lernen, in: Achtenhagen, F. \& Lempert, W. (Hrsg.): Lebenslanges Lernen - seine Grundlegung im Kindes- und Jugendalter, Band 4: Formen und Inhalte von Lernprozessen, Opladen: Leske + Budrich, S. 6090.

Sembill, D. (2004): Abschlussbericht zu "Prozessanalysen Selbstorganisierten Lernens" im Rahmen des DFG-Schwerpunktprogramms "Lehr-Lern-Prozesse in der kaufmännischen Erstausbildung". Bamberg. Download unter http://wipaed.sowi.uni-bamberg.de/awgs/ wipaed2002/download-pdf/abschlussbericht_prozess-analysen.pd

Sembill, D., Wolf, K. D., Wuttke, E., Santjer, I. \& Schumacher, L. (1998): Prozessanalysen Selbstorganisierten Lernens, in: Beck, K. \& Dubs, R. (Hrsg.): Kompetenzerwerb in der Berufserziehung. Kognitive, motivationale und moralische Dimensionen kaufmännische Qualifizierungsprozesse, in: Zeitschrift für Berufs- und Wirtschaftspädagogik, Beiheft $\mathrm{Nr}$ 14, Stutigart: Franz Steiner, S. 57-79.

Sembill, D. Schumacher, L., Wolf, K. D., Wuttke, E. \& Santjer-Schnabel, I. (2001): Förderung der Problemlösefähigkeit und der Motivation durch Selbstorganisiertes Lernen, in Beck, K. \& Krumm, V. (Hrsg.): Lehren und Lernen in der beruflichen Erstausbildung Grundlagen einer modernen kaufmănnischen Berulsqualifizierung, Opladen: Leske Budrich, S. 257-281.

Sembill, D., Wolf, K. D., Wuttke, E. \& Schumacher, L. (2002): Self-organized learning in vocational education - Foundation, implementation, and evaluation, in: Beck, K. (Ed.) Teaching-fearning processes in vocational education, Frankfurt/Main u. a.: Lang, pp. 267 295 
Wenger, E. C. (1998): Communities of practice - Learning, meaning, and identity. (Cambridge University Press) Cambridge.

Wenger, E. C., McDermott, R. \& Snyder, W. M. (2002): Cultivating Communities of Practice (Harvard Business School Press) Boston, Massachusetts.

Wolf, K. D., Städtler, H. \& Baumann, R. (2002): Authentische Lernsituationen - Best Practice virtueller Lehrangebote am Beispiel Columbia und Cardean University. Vortragsfolien, http://www.vhb. org/dokumente/workshops/ws05072002/vhbDid WorkshopWolf.pdf.

Wolf, K. D. (2003): Gestaltung und Einsatz einer internetbasierten Lernumgebung zur Unterstützung selbstorganisierten Lernens, Hamburg: Dr. Kovač.

Wolf, K. D. (2004): Fragebogen Computer-Vorwissen und -Interesse (CoVl). Bamberg: Unveröffentlichter Fragebogen. 